\title{
Perinatal death recording: time for a change?
}

\author{
MAUREEN J SCOTT, J W K RITCHIE, B G MCCLURE, M MCC REID, H L HALLIDAY
}

\begin{abstract}
The new perinatal death certificate proposed by the World Health Organisation was examined in relation to existing measures for recording perinatal death statistics and also with regard to new information gathered. Present procedures appear to underestimate the number of perinatal deaths by roughly $10 \%$, though late registrations may lower this figure slightly. The use of a minimum birth weight as the criterion for inclusion in perinatal statistics removed much of the uncertainty associated with definitions of live birth and stillbirth. The new certificate led to duplication of some information already recorded through birth notification yet failed to provide information on some other factors generally considered relevant to perinatal mortality. The format proposed for recording cause of death provided a more logical presentation of events.

Standardising birth information recorded on all infants, modifying death certificates, and developing efficient record-linkage schemes would be more valuable than introducing the WHO certificate. Useful interpretation of the meaning of the characteristics of infants dying in the perinatal period awaits these timely changes.
\end{abstract}

\footnotetext{
Queen's University of Belfast, Institute of Clinical Science, Belfast BT12 6BJ

MAUREEN J SCOTT, MD, lecturer, department of community medicine

J W K RITCHIE, MD, MRCOG, senior lecturer, department of midwifery and gynaecology

B G MCCLURE, FRCP, DCH, senior lecturer in child health
}

Royal Maternity Hospital and Belfast City Hospital, Belfast M MCC REID, FRCP, DCH, consultant neonatologist

H L HALLIDAY, MD, MRCP, consultant neonatologist

\section{Introduction}

In Geneva in October 1975 the International Conference for the Ninth Revision of the International Classification of Diseases convened by the World Health Organisation considered the report of the Scientific Group on Health Statistics Methodology relating to perinatal events and also the recommendations of the expert committee. The conference recommended that, "where practicable statistics in relation to perinatal deaths should be derived from a special certificate of perinatal death (instead of the normal death certificate)"' presenting such information (fig 1).

Observed differences in current perinatal mortality rates among various countries have been used to measure the standards of perinatal care and the effectiveness of social and public health measures. ${ }^{2}$ The value of crude perinatal mortality statistics for such purposes is questionable because of variations in laws and customs relating to the registration of births and deaths and the lack of information concerning the distribution of births by birth weight and malformation rates in the populations studied.

Criteria for inclusion in perinatal statistics have elements of uncertainty. The use of a gestational age of 28 weeks as a cut-off point to define late fetal deaths does not allow that maturity is uncertain in a considerable number of pregnancies, perhaps especially so in those that end in early death. Chalmers and Mcfarlane ${ }^{3}$ have pointed out that the definition of live birth applied at different gestational ages may be affected by factors such as place of delivery, rules relating to payment of maternity and other statutory financial benefits, and the religious and cultural affiliations of the community.

The World Health Organisation, in proposing the introduction of a new perinatal death certificate, suggested adopting more objective criteria and recommended, "that national perinatal statistics should include all fetuses and infants delivered weighing at least $500 \mathrm{~g}$ (or, when birth weight is unavailable, the corresponding gestational age (22 weeks) or body length $(25 \mathrm{~cm}$ crown-heel)) whether alive or dead."1 Northern Ireland has had 
a relatively high perinatal mortality rate, comparing unfavourably with many other countries. ${ }^{4}$ We decided to use the proposed new perinatal death certificate for one year (in parallel with existing statutory procedures for recording stillbirth and death) to examine the completeness of existing procedures and also to assess the usefulness of the proposed new document.

\section{Materials and methods}

At a meeting in November 1978, at which the findings of a previous study ${ }^{5}$ were presented, copies of the proposed new perinatal death certificate (fig 1) were distributed with a request for completion of the new certificate for all perinatal deaths during 1979. One of us (MJS)

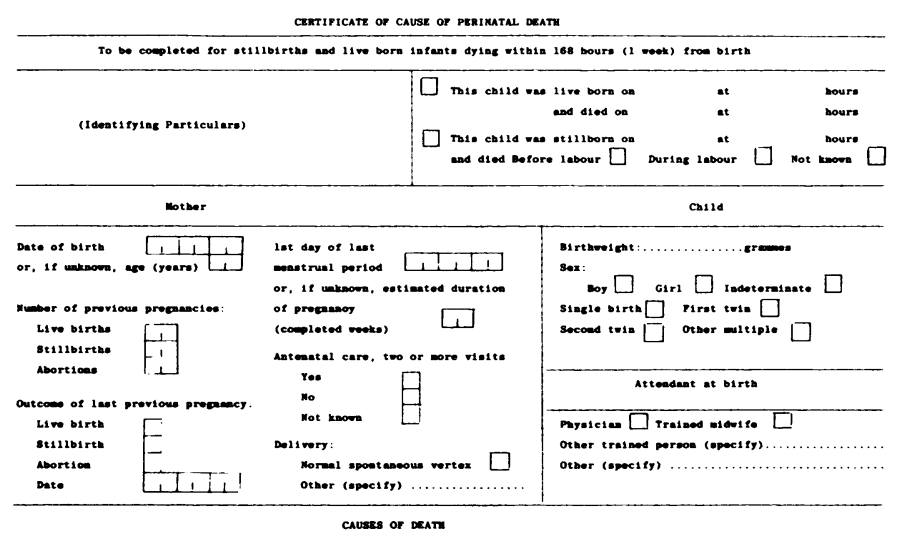

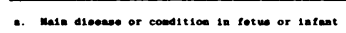

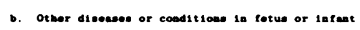

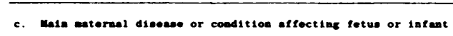

- Othor rolevant circuentasces

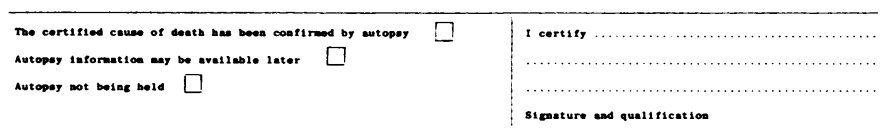

FIG $1-$ Certificate of cause of perinatal death.

then sent copies to all maternity units, along with a personal letter to all consultants and senior midwifery staff, indicating clearly the criteria for inclusion and offering guidance on completing the certificate. Information from completed certificates was transferred to punch cards for subsequent analysis.

With the co-operation of the General Register Office we examined stillbirth and death registrations for infants born during 1979, and transferred this information to punch cards for analysis. Infants for whom we received perinatal death certificates were "matched" with the statutory death registrations and information from these two sources was compared.

\section{Results}

In Northern Ireland in 1979 births in hospitals numbered $28102 .^{\circ}$ Domiciliary births have recently been $0.5 \%$ of all births, so that a total of 28250 births seems a reasonable estimate. Figure 2 shows the numbers of perinatal deaths recorded by the Registrar General and those reported to us on WHO perinatal death certificates. In 1979 the Registrar General's death registrations showed 237 stillbirths and 203 who died within seven days-a total of 440 perinatal deaths. All stillbirths registered had a gestational age greater than 28 weeks. Although birth weight and gestational age are not officially recorded on death certificates they were occasionally recorded in parentheses, especially where prematurity was noted as a cause of death, and clearly some infants having a gestational age as low as 20 weeks or a birth weight of around $400 \mathrm{~g}$ were included in death registrations relating to infants who had been designated live born.

We received 413 perinatal death certificates from 19 maternity units relating to infants born weighing over $500 \mathrm{~g}$ or, in the 12 cases where birth weight was not recorded, of gestational age greater than 22 weeks. Some 194 infants were live born and 219 stillborn, and 344 of these were matched to deaths recorded by the Registrar General. Inspection of the official registrations relating to the 96 registered perinatal deaths for whom we did not receive perinatal death certificates showed that 11 infants weighed under $550 \mathrm{~g}$ and would therefore not have required perinatal death certificates according to the WHO definition issued to those taking part in the study. This may be the explanation for some others but birthweight is not routinely recorded on current death certificates. Some 16 registered deaths occurred in a unit that, having initially agreed to take part in the study, ceased sending us certificates after the first couple of months, and eight deaths occurred at home. Almost all participating units occasionally failed to return certificates, especially in July and August.

We received perinatal death certificates for 69 infants weighing over $500 \mathrm{~g}$ and we could find no trace of official registration by the end of April 1980. Contrary to our expectation that these infants would be mostly stillbirths of less than 28 weeks' gestation and weighing more than $500 \mathrm{~g}$ only 22 of the infants were in this group. Sixteen were stillbirths of more than 28 weeks' gestation and 31 were live births. Therefore 47 deaths that according to existing legislation should have been registered with the Registrar General were not found in our search of death registrations (fig 2).

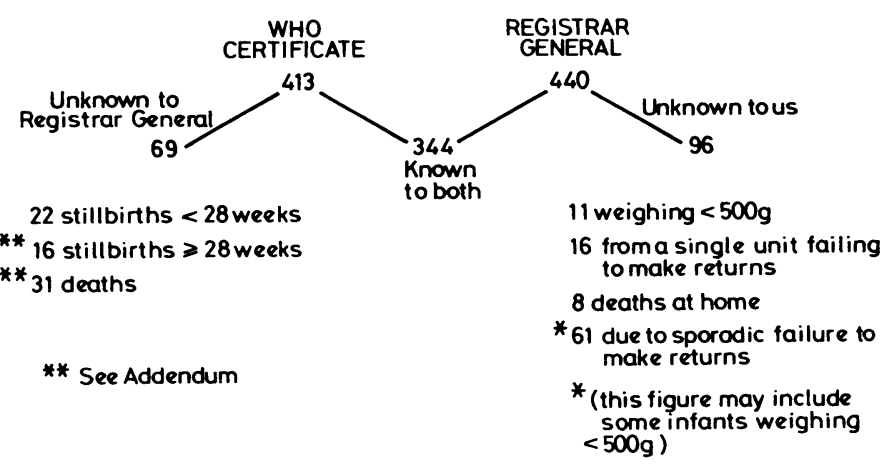

FIG 2-Comparison of perinatal deaths from WHO certificates and Registrar General's records.

We considered the proposed new perinatal death certificate in relation to information already recorded about perinatal death. Much more information is required on the new certificate than on the certificate of stillbirth (appendix 1) or on the medical certificate of cause of death (appendix 2). Most of these new questions were answered in more than $95 \%$ of cases, exceptions being questions relating to mother's date of birth $(86.7 \%)$, date of last menstrual period $(74 \%)$, date of completion of last pregnancy $(68.6 \%)$, and antenatal care $(93 \%)$. Birth weight was not recorded in 12 cases, but for each of these the gestational age was known to exceed 22 weeks, and the time of birth was unrecorded in 14 stillbirths. Because of incomplete answers regarding date, time of birth, or death it was impossible to estimate precise age at death in 12 live-born infants.

In one case no main cause of death was recorded on the new certificate, presumably owing to an oversight on the part of the doctor completing the certificate. In 167 cases we obtained information about other diseases or conditions of the infant, and in 163 at least one maternal condition affecting the infant was recorded. Some 33 women had a second condition affecting the infant, and in 65 cases we received information about other circumstances relevant to the death. The new certificate provided us with a total of 840 items of information about causes of death in 413 infants. The Registrar General's records showed 775 items of information on the causes of death in 440 infants.

In only 202 of the 344 cases where matching of our certificates with the Registrar General's was possible was the leading cause of death identical. In 59 the same two conditions appeared in leading and antecedent causes but the order of presentation was reversed. In a further 37 the leading cause noted on the statutory certificate appeared under "other disease in infant" on our certificate and our leading 
cause was not mentioned on the registered certificate; this was most commonly found where "prematurity" was the leading cause registered. In 22 cases a general condition recorded by the Registrar General-for instance, multiple malformations-was replaced by a more precise diagnosis on our certificate, and in 24 the diagnoses recorded by the two sources were completely different.

\section{Discussion}

We have examined the existing measures for recording perinatal mortality; and the observed difference in 1979 between the registered perinatal deaths and perinatal deaths reported to us requires explanation.

A small discrepancy is inevitable since some days may elapse between death and registration. Deaths occurring at the end of December may not be registered until January and of the 47 deaths that we were unable to match, 13 occurred after 15 December 1979. Death must be registered within five days of the event or, if preliminary notice of death has been given to the registrar, within 14 days. " If a body is disposed of without the proper registration procedure having been completed then the person who effects such disposal must give notice of this to the registrar within seven days. The registrar may register a death up to 12 months after the event, or a stillbirth up to three months. Although he attempts to inform himself of all deaths in his district, late registrations do occur. Our first search of the records ${ }^{8}$ showed no registrations relating to 85 perinatal deaths but 24 were subsequently found to have been late registrations. Information for a further five cases was with the coroner's office and we had received duplicate perinatal death certificates for another nine.

There may be under-registration of perinatal death of about $10 \%$ in Northern Ireland. Published statistics refer to events registered in any year so they reflect the degree to which the law relating to registration is observed. If the 34 deaths that occurred before 15 December had been registered within the statutory time the perinatal death rate for 1979 births would be increased by $1 \cdot 2$.

Perinatal mortality figures are also influenced by how obstetric staff interpret the definition of live birth. Published statistics in Northern Ireland include infants weighing below $400 \mathrm{~g}$ and of maturity as low as 20 weeks which elsewhere might have been considered abortions. The definitions suggested in the new WHO certificate would do much to remove this uncertainty.

Current requirements for registration may also lead to some confusion in interpreting the outcome of twin pregnancies. Where delivery occurred before 28 weeks' gestation and resulted in a liveborn and a stillborn infant only the liveborn twin would appear in official figures. This anomaly could still arise under the proposed new definitions if one twin weighed under $500 \mathrm{~g}$.

Our second aim was to look at the usefulness of the proposed new certificate. At present a considerable amount of information is required from the informant (usually a relative) at registration of a stillbirth or death but the information requested is not identical for the two groups. The new WHO certificate standardises the information recorded on perinatal deaths and also obtains it from a more reliable source. With virtually $100 \%$ hospital confinement obstetric notes should be readily available. Recording the increased amount of information required on the new certificate increases the work of obstetric staff, but if a duplicate copy were retained by each unit it would allow simple and rapid audit of the perinatal mortality there.

In Northern Ireland it proved difficult to get complete answers to questions relating to mother's date of birth, date of last menstrual period, and date of completion of last pregnancy. The recording of the "date of completion of last previous pregnancy" allows estimation of inter-pregnancy interval, but we would question the usefulness of recording this interval or indeed recording the "outcome of last previous pregnancy" in the absence of more detailed information about the cause of a previous unsatisfactory outcome.
The question regarding "number of antenatal visits" proved difficult to answer and interpret correctly since care was often carried out by the general practitioner, hospital staff, and community midwives or a combination of these. The mothers' notes may record only hospital visits, which in cases of shared antenatal care may start regularly only in the last trimester. Incorrect conclusions may be drawn since so many babies dying in the perinatal period are of low gestational age that mothers may not have had the chance to make frequent visits before delivery supervened.

Illegitimacy and low social class are considered as adverse factors for perinatal survival, ${ }^{9}$ and perhaps surprisingly the new WHO perinatal death certificate does not include questions on father's age and occupation, marital state of mother, and duration of marriage. Place of birth and place of death are not specifically requested on the proposed new certificate. We think it important that this information should be included.

We welcomed the facility provided by the new certificate to separate stillbirths into those occurring before labour and during labour.

Matching the information recorded about cause of death on the new certificate to that recorded on the statutory certificate showed that in $65 \%$ of cases the leading cause was identical. We would have expected it to be similar in all cases, since both were being completed by the same person. We considered that the new certificate encouraged a more logical presentation of the sequence of events leading to death and also permitted a fuller account of the events. The extra information recorded on the proposed new certificate related in almost all cases to conditions in the mother or placental or cord conditions.

Incomplete registration of deaths would still be a problem if the proposed new WHO certificate were introduced. It might be overcome by showing the mother the perinatal death certificate which might incorporate a space where she could sign that the particulars recorded are correct to the best of her knowledge. Each unit could then give the mother a copy to retain and send the original certificate directly to the Registrar General, in the same way as birth notifications are forwarded to local health and social services boards.

Nevertheless, despite the advantages of defining the perinatal death population more precisely and the increased information provided by the proposed new certificate, we would question the usefulness of such a document in epidemiological research. The "population at risk" is a basic concept for medical research, and the definition of this population must begin with some basic characteristic common to all members. If observations made on a particular subgroup are not related to all persons at risk then any conclusions may be of little value. As every birth is at risk of being a perinatal death, studies of deaths alone without relating these to the total birth population are of limited use. A more valuable approach to studying perinatal death would be through urgent modification of existing procedures for recording information and the development of efficient record-linkage schemes. If the latter were developed birth notification forms should be designed to record basic biosocial information on all births, and death certificates need then record only place of death, age at death, and causes of death. Stillbirths might be documented on a separate form identical to the new birth notification form but with an added facility to record the cause of death. This approach would provide basic data common to all births, live and still; would avoid duplication of information; and would allow the study of death at any age postnatally.

We conclude that the information currently collected on perinatal death needs reorganising to be of value. In the absence of comparable total birth population data we do not think the proposed new WHO perinatal death certificates would serve a useful epidemiological purpose.

We express our sincere thanks to the medical and nursing staffs of the maternity units in Northern Ireland, without whose co-operation this study could not have been undertaken. We are also indebted to Mrs Margaret Bowker and Mrs Iris Hay and her staff for help in 
collecting and preparing data for analysis. We also thank the staff of the General Register Office in Belfast and the secretarial staff of the Department of Community Medicine, Queen's University, Belfast.

We have received financial support for this work from the Northern Ireland Council for Orthopaedic Development and the Department of Health and Social Services. We express our appreciation of their interest and help.

\section{ADDENDUM}

This article was prepared for publication in October 1980 and reports the situation with regard to 1979 registration of perinatal death as known to us at that time. I am now informed by the General Register Office that registrations relating to 13 of the 16 stillbirths and 26 of the 31 deaths that we had initially been unable to match have now been traced in their records.

\section{References}

1 World Health Organisation. Manual of the international statistical classification of diseases, injuries and causes of death. 9th revision. Vol 1. Geneva: WHO, 1977

${ }^{2}$ Alberman E. Perinatal mortality rates. $B r \mathcal{F}$ Hosp Med 1978 ;20:439-43.

- Chalmers I, McFarlane A. Interpretation of perinatal statistics. In: Wharton BA, ed. Topics in perinatal medicine. Tunbridge Wells: Pitman Medical, 1980

4 Department of Health and Social Security. On the state of the public health for the year 1977. London: HMSO, 1978.

s Scott MJ, McClure G, Reid M, Ritchie JWK, Keilty SR. Neonatal death in Northern Ireland. Br Med $\mathcal{F} 1978$;ii:987-9.

- Registrar General. Quarterly return for Northern Ireland, quarter ended 31 st December 1979 (also preliminary return for the year 1979). Belfast: HMSO, 1980.

7 Births and Deaths Registration Act (NI). Belfast: HMSO, 1967.

${ }^{8}$ Chalmer I, McIlwaine G, eds. Perinatal audit and surveillance. London: Royal College of Obstetricians and Gynaecologist (in press).

- Committee on Child Health Services. Fit for the future. London: HMSO, 1976.

(Accepted 21 November 1990)

\section{Appendix 1}

INFORMATION ON PROPOSED WHO PERINATAL CERTIFICATE NOT ON 1 Place of birth.

$$
\text { EXISTING STILLBIRTH CERTIFICATE }
$$

2 Time of stillbirth. Before/during labour. Not known.

$3^{*}$ Number of previous pregnancies-live births, ${ }^{*}$ stillbirths, * abortions.
4 Outcome of last previous pregnancy-live birth, stillbirth, abortion.

5 Date of completion of last previous pregnancy.

6 First day of last menstrual period.

7 Antenatal care, $<2$ visits, $\geqslant 2$ visits, not known.

8 Delivery-normal spontaneous vertex, other (specify).

9* Multiplicity of birth-single birth, first twin, second twin, other multiple.

10 Attendant at birth-doctor, trained midwife, other trained person (specify), other (specify).

$11+$ Causes of death:

(a) Main disease or condition in fetus or infant.

(b) Other diseases or conditions in fetus or infant.

(c) Main maternal disease or condition affecting fetus or infant.

(d) Other maternal disease or conditions affecting fetus or infant.

(e) Other relevant circumstances.

* Total number of previous livebirths and stillbirths to mother and whether child was offspring of a multiple pregnancy are currently required information on stillbirth registration.

$\dagger$ These questions are asked in less precise form on existing certificate of stillbirth.

\section{Appendix 2}

INFORMATION ON PROPOSED WHO PERINATAL DEATH CERTIFICATE NOT ON EXISTING MEDICAL CERTIFICATE OF CAUSE OF DEATH

1 Date of birth.

2 Time of birth.

3 Time of death.

4 Mother's date of birth.

5 Mother's age.

6 Number of previous pregnancies-live births, stillbirths, abortions.

7 Outcome of last previous pregnancy-live birth, stillbirth, abortion.

8 Date of completion of last previous pregnancy.

9 Birth weight.

10* Sex.

11 Multiplicity of birth-single birth, first twin, second twin, other multiple.

12 Attendant at birth-doctor, trained midwife, other trained person (specify), other (specify).

13 Causes of death.

(a) Main disease or condition in fetus or infant.

(b) Other diseases or conditions in fetus or infant.

(c) Main maternal disease or condition affecting fetus or infant.

(d) Other maternal diseases or conditions affecting fetus or infant.

(e) Other relevant circumstances.

14 Necropsy done/may be done/will not be done.

* This is currently required information on death registration.
Now that it has been suggested that oil of bergamot may be carcinogenic when applied to the skin (in suntan lotions), is there likely to be a similar danger in ingesting china tea flavoured with bergamot, such as Earl Grey?

Bergamot oil contains 5-methoxypsoralen (5-MOP), which enhances the carcinogenic effect of ultraviolet light (within a critical wave-band) on the skin of mice. There is no evidence that bergamot oil by itself causes cancer of the skin. Theoretically, ingested 5-MOP might enhance ultraviolet skin carcinogenesis, but its concentration in bergamot tea is far too low for any concern.

What is the calorific content per gramme of bran eaten to supply roughage?

Wheat bran does not have a constant composition. The amount of starchy endosperm associated with a bran depends on the method used to grind the wheat and the efficiency with which the bran has been sieved. A typical bran ${ }^{1}$ separate from wholewheat grain in a roller mill will provide about $206 \mathrm{kcal} / 100 \mathrm{mg}$. A bran prepared by sieving wholemeal flour usually contains a little more starch and, therefore, has a slightly higher energy value, around $222 \mathrm{kcal} / 100 \mathrm{~g}$. These energy values are calculated from the composition of the products, using calorie conversion factors ${ }^{2}$ that discount any energy derived from the fatty acids produced by fermentation of dietary fibre in the large bowel. In the case of bran this contribution is quite small, and as the dietary fibre usually leads to small increases in the faecal losses of nitrogen and fat, the use of the conventional calorie values is quite adequate for most dietetic purposes.

'Paul AA, Southgate DAT. McCance and Widdowson's-the composition of foods. 4th ed. London; HMSO, 1978.

Southgate DAT, Durnin JVGA. Calorie conversion factors. An experimental reassessment of the factors used in the calculation of the energy value of human diets. Br $\mathcal{F}$ Nutr 1970;24:517-35.

What is the mechanism of pain in "heartburn" of gastric origin. Is it primarily due to reflux acid on the oesophageal mucosa or to spasm or distension of the gastro-oesophageal sphincter?

Heartburn is usually caused by irritation of a sensitive oesophageal mucosa by refluxed gastric contents or swallowed material. Thus some sufferers experience pain as they swallow hot fluid or alcohol, and the symptoms may be reproduced by dripping hydrochloric acid into the gullet (Bernstein test). Changes in motility are sometimes observed during such pain and may increase it, but such "spasm" is not usually the primary mechanism. 